Article

\title{
Study of Osteocyte Behavior by High-Resolution Intravital Imaging Following Photo-Induced Ischemia
}

\author{
Hengfeng Yuan ${ }^{1,2}$, Wen Jiang ${ }^{3}$, Yuanxin Chen ${ }^{1, *}$ and Betty Y. S. Kim ${ }^{1}$ \\ 1 Department of Neurosurgery, Mayo Clinic College of Medicine, 4500 San Pablo road, Jacksonville, FL 32224, \\ USA; yuanfengfengggg@163.com (H.Y.); kim.betty@mayo.edu (B.Y.S.K.) \\ 2 Shanghai Medical Schools, Fudan University, 138 Yixueyuan Road, Xuhui, Shanghai 200032, China \\ 3 Department of Radiation Oncology, UT Southwestern Medical Center, 6001 Forest Park Road, Dallas, \\ TX 75235, USA; jiangwen0215@gmail.com \\ * Correspondence: chen.yuanxin@mayo.edu
}

Academic Editors: Lesheng Teng, Zhaogang Yang and Jing Xie

Received: 14 September 2018; Accepted: 28 October 2018; Published: 4 November 2018

check for updates

\begin{abstract}
Ischemic injuries and local hypoxia can result in osteocytes dysfunction and play a key role in the pathogenesis of avascular osteonecrosis. Conventional imaging techniques including magnetic resonance imaging (MRI) and computed tomography (CT) can reveal structural and functional changes within bony anatomy; however, characterization of osteocyte behavioral dynamics in the setting of osteonecrosis at the single cell resolution is limited. Here, we demonstrate an optical approach to study real-time osteocyte functions in vivo. Using nicotinamide adenine dinucleotide (NADH) as a biomarker for metabolic dynamics in osteocytes, we showed that NADH level within osteocytes transiently increase significantly after local ischemia through non-invasive photo-induced thrombosis of afferent arterioles followed by a steady decline. Our study presents a non-invasive optical approach to study osteocyte behavior through the modulation of local environmental conditions. Thus it provides a powerful toolkit to study cellular processes involved in bone pathologies in vivo.
\end{abstract}

Keywords: osteocyte; osteonecrosis; two-photon; NADH

\section{Introduction}

Avascular osteonecrosis (AVN), commonly found in the regions of femoral head, is associated with bony cell death. Although AVN is attributed to ischemic insults, the exact molecular mechanism remains controversial [1,2]. Previous studies have suggested that intravascular thrombus and extravascular marrow lipid deposition can result in impaired blood supply leading to regional ischemia. The decreased oxygen tension ultimately causes osteocyte death and eventual collapse of subchondral bones [3,4]. Modern imaging techniques, such as magnetic resonance imaging (MRI), Single-photon emission computerized tomography (SPECT) and positron emission tomography (PET), have been utilized extensively in clinical settings to diagnose AVN [5-8]. However, imaging-based approaches that rely on optical platforms to study the cellular and molecular pathogenesis of AVN, possess unique technical challenges due to the high photon attenuation of calcified bone matrix.

Our current study aims to overcome many of the challenges associated with optical-based imaging of bony compartments and elucidates a potential molecular pathophysiology of AVN. Nicotinamide adenine dinucleotide (NADH), an important metabolic coenzyme in oxidative phosphorylation $[9,10]$, is a sensitive non-linear proxy of tissue hypoxia [11,12]. Moreover, NADH is also a cofactor used by fatty acid synthetase in lipid biosynthesis, and by enzymes such as desmolases and hydroxylases in steroid biosynthesis [13]. NADH level within cells thus provides an accurate 
reflection of their metabolic demand and may serve as a surrogate marker for functional changes within osteocyte in AVN.

Due to the unique auto-fluorescence properties of NADH, imaging of NADH to monitor cellular metabolic states in vivo has been investigated in the past [14-16]. Recent studies have demonstrated that two-photon excitation microscopy may further enhance NADH imaging in vivo [16,17]. Using longer wavelength photons to excite NADH, less tissue scattering is expected, which will result in improved signal detection with reduced background noise. The small focal excitation volume further improves imaging resolution in the axial and longitudinal plane, thus enabling single cell imaging and tracking in deep tissue compartments such as the bone, marrow cavity or soft tissues [18-21].

Utilizing these advantages, our current study proposes a novel, all optical-based approach to generate and study the hypoxic microenvironment of AVN in situ. Performing high resolution real-time and long-term imaging of necrotic processes occurring in mouse calvaria, our goal is to characterize the deterministic relationships between vascular occlusion, osteocyte death, and changes in intracellular metabolic demand that contribute to the development of AVN.

\section{Results}

\subsection{Real-Time In Vivo Imaging of Osteocyte Structure and Blood Vessels}

To track and visualize the live osteocytes and vasculature in the bone, a common dye, Calcein AM, was subcutaneously administrated (S.C, $20 \mathrm{mg} / \mathrm{kg}$ ) 3 days before imaging and the dextran (70 kDa Texas red) intravenously injected 5 minutes before imaging (I.V, $10 \mathrm{mg} / \mathrm{kg}$ ), Figure 1 showed the structure of the live osteocytes (green) and bone vasculature (red) using two-photon imaging system. We found that the live osteocytes were distributed and clustered along the vasculature (Supplementary Movie 1: Live osteocytes were distributed and clustered along the vasculature). In order to further confirm that the observed area was limited to the bone, we magnified the image area to visualize the osteocytes and extracellular matrix component within the bone (Figure 2). We observed that osteocytes were connected to one another through a network of cytoplasmic projections, including disk-shaped osteocytic lacunae and an abundance of dendritic canaliculus. Furthermore, the skull had been removed and the cortex exposed. The image from the cortex showed no labeled cells with Calcein dye (data not shown), which indicated that Calcien was specific to target and label osterocytes and imaging area did not exceed the range of bone.

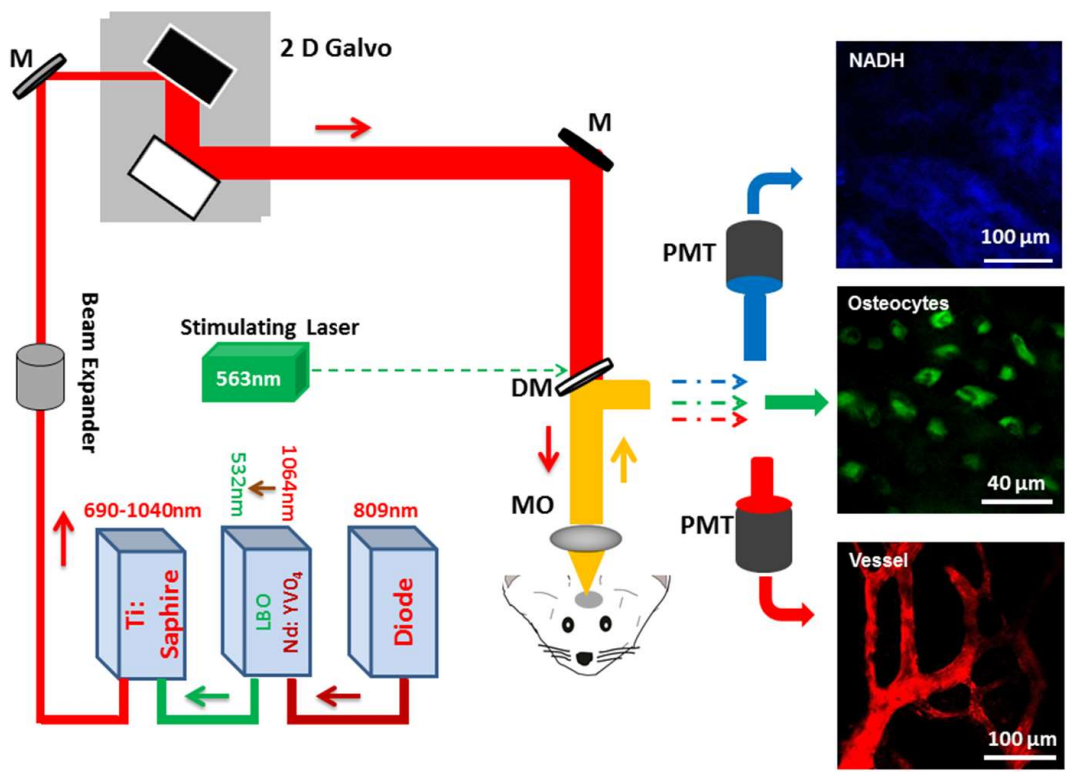

Figure 1. Schematic illustration of two photon imaging system. Abbrv: M, mirror; DC, dichroic mirror; $\mathrm{MO}$, microscope objective; PMT, photomultiplier tube; 2D Galvo, two dimension galvanometer 


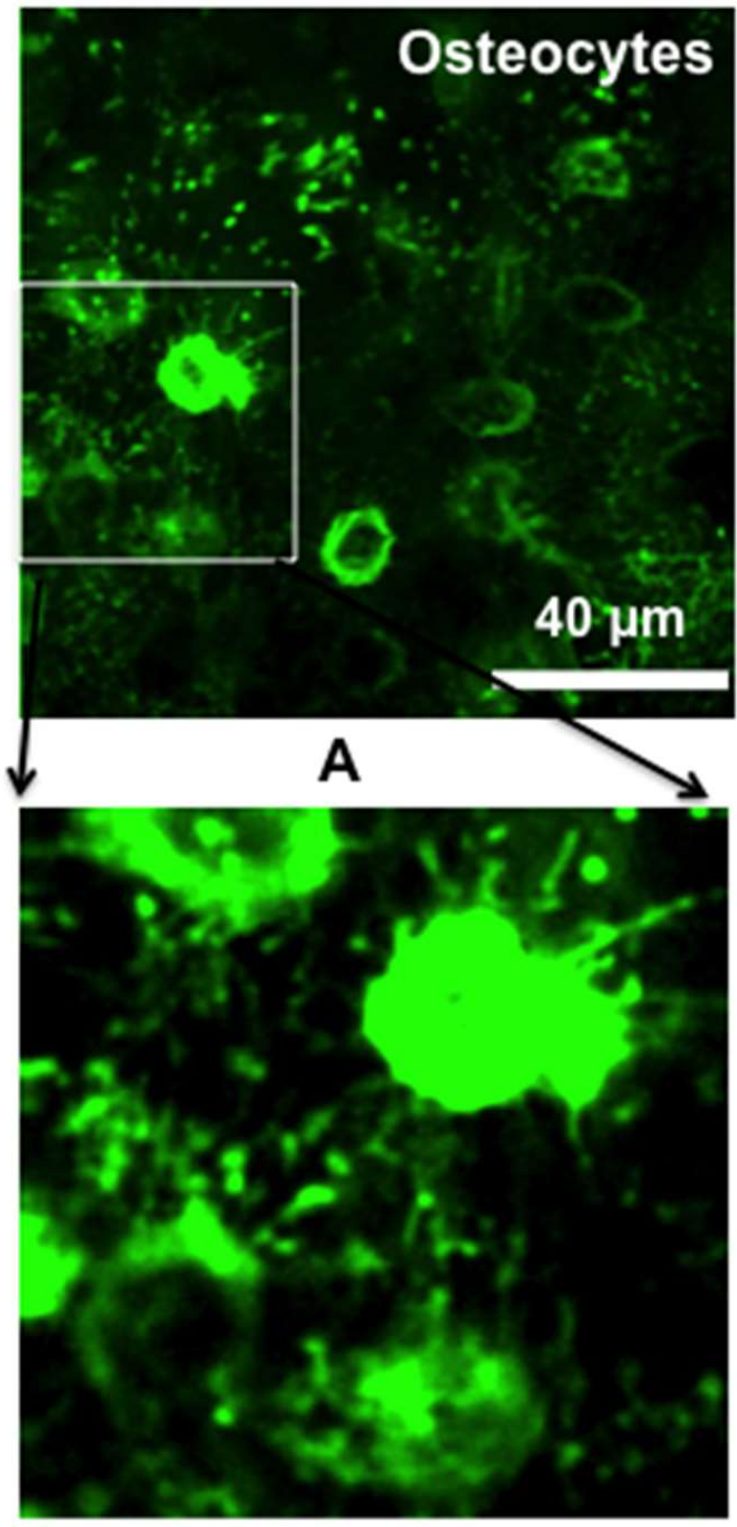

C

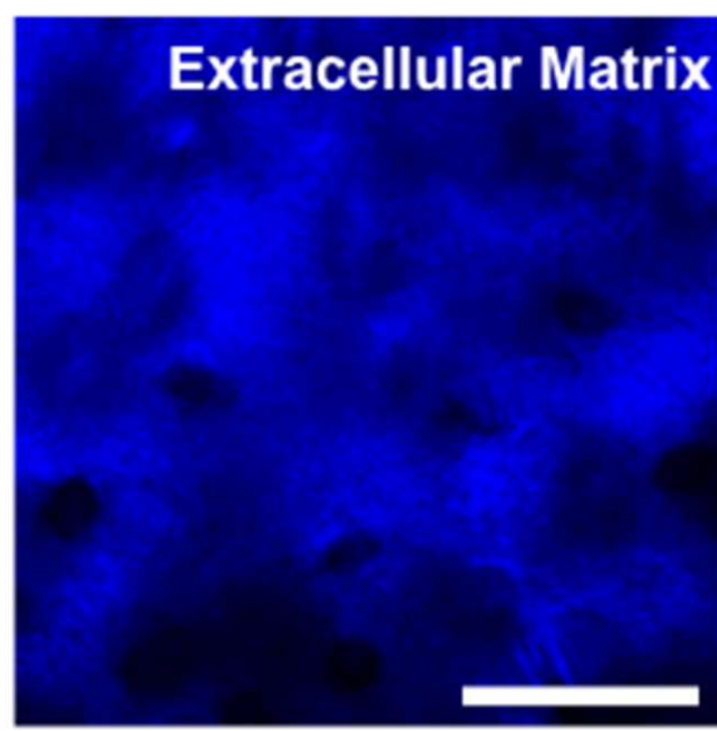

B

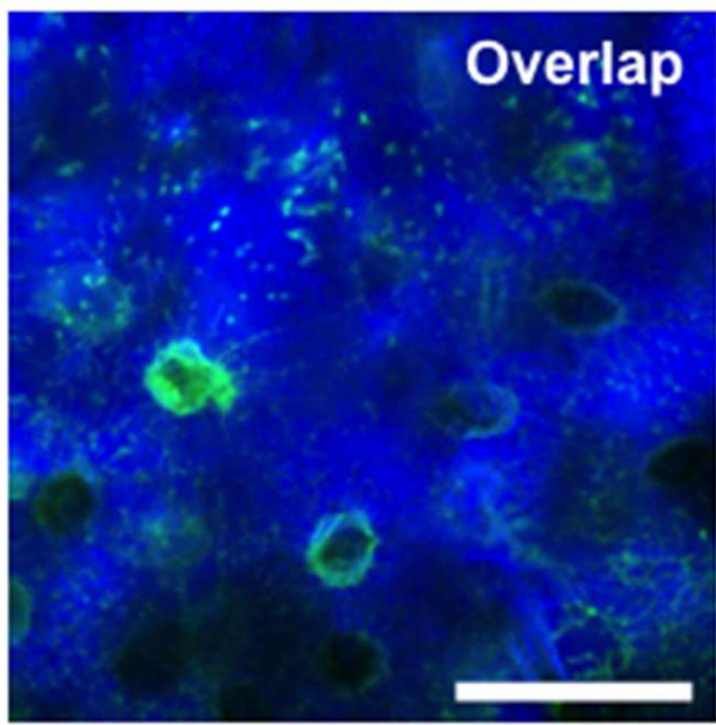

D

Figure 2. Intravital two-photon imaging of osteocytes: (A) Osteocytes were connected to one another through a network of cytoplasmic projections; (B) Extracellular matrix; (C) Magnification of the inset in Figure 2A; (D) Overlap of Figure 2A,B.

\subsection{Photo-thrombosis of the Selected Blood Vessels}

To investigate the effects of blood flow supply on osteocytes metabolism, laser beam (563 nm) was used to target and stimulate the arterioles (white circle in Figure 3A) to make photo-thrombosis ischemic models after the intravenously administration of photosensitive dye, Rose Bengal (RB, I.V). After photo-stimulation, many black dots (un-labeled RBC) were found within the vessel (Figure 3B) with a reduction in blood flow and in some instances completely halted with the velocity under $0.2 \mathrm{~mm} / \mathrm{s}$ (Figure 3C, Supplementary Movies 2 and 3: blood flow before and after photo-thrombosis, respectively). 


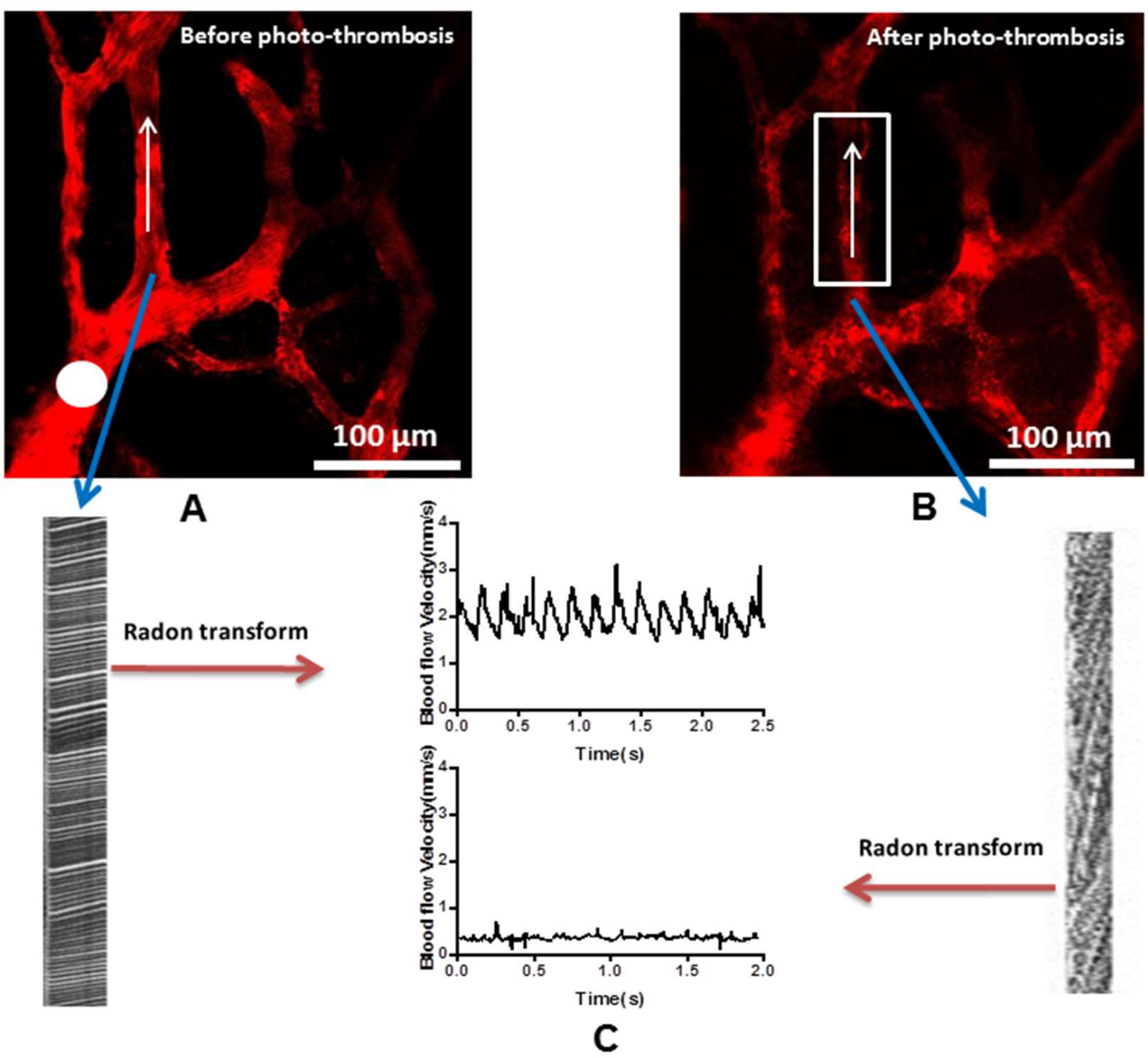

Figure 3. Photo-thrombosis of the arterioles: (A) Before photo-thrombosis, white circle demonstrates the target site of the laser beam; (B) after photo-thrombosis, many black dots (un-labeled RBC) were noted within the vessel; $(\mathbf{C})$ velocity measurement of the blood flow.

\subsection{In Vivo Quantitation of Osteocyte Metabolism}

To study the changes of osteocytes metabolism under the ischemic and hypoxic microenvironment, propidium iodide (PI, sigma-aldrich, St. Louis, Missouri, USA; $1 \mathrm{mg} / \mathrm{ml}$ ) was intravenously administered to track the live cell state. We took images (shown in Figure 4A) at different time points ( 0,3 and $6 \mathrm{~h}$ after photo-thrombosis). Our data indicated that before the photo-thrombosis, only a few stained cells with PI dye were captured (percentage $<10 \%$ ) (Figure $4 \mathrm{~B}$ ), which might be caused by the toxicity of staining dyes. Three hours after complete blood supply deprivation following photo-thrombosis, the percentage of PI stained dead cells were increased to approximately $75 \%$, and fluorescence intensity ratio of PI/Calcein achieved 0.29 (Figure 4C). After $6 \mathrm{~h}$, the dead cell rates reached nearly $95 \%$ and a PI/Calcein ratio of 2.31. In addition, the cell structure expanded due to cells lysis (Figure 4A, $6 \mathrm{~h}$ ).

Simultaneously, NADH auto-fluorescence inside the bone had been collected under $740 \mathrm{~nm}$ laser beam excitation (Figure 5A-D). The NADH signal increased $30 \mathrm{~min}$ after the photo-thrombosis initial response to NADH accumulation caused by ischemia and hypoxia but decreased afterwards due to blood flow reperfusion (Figure 5E). 

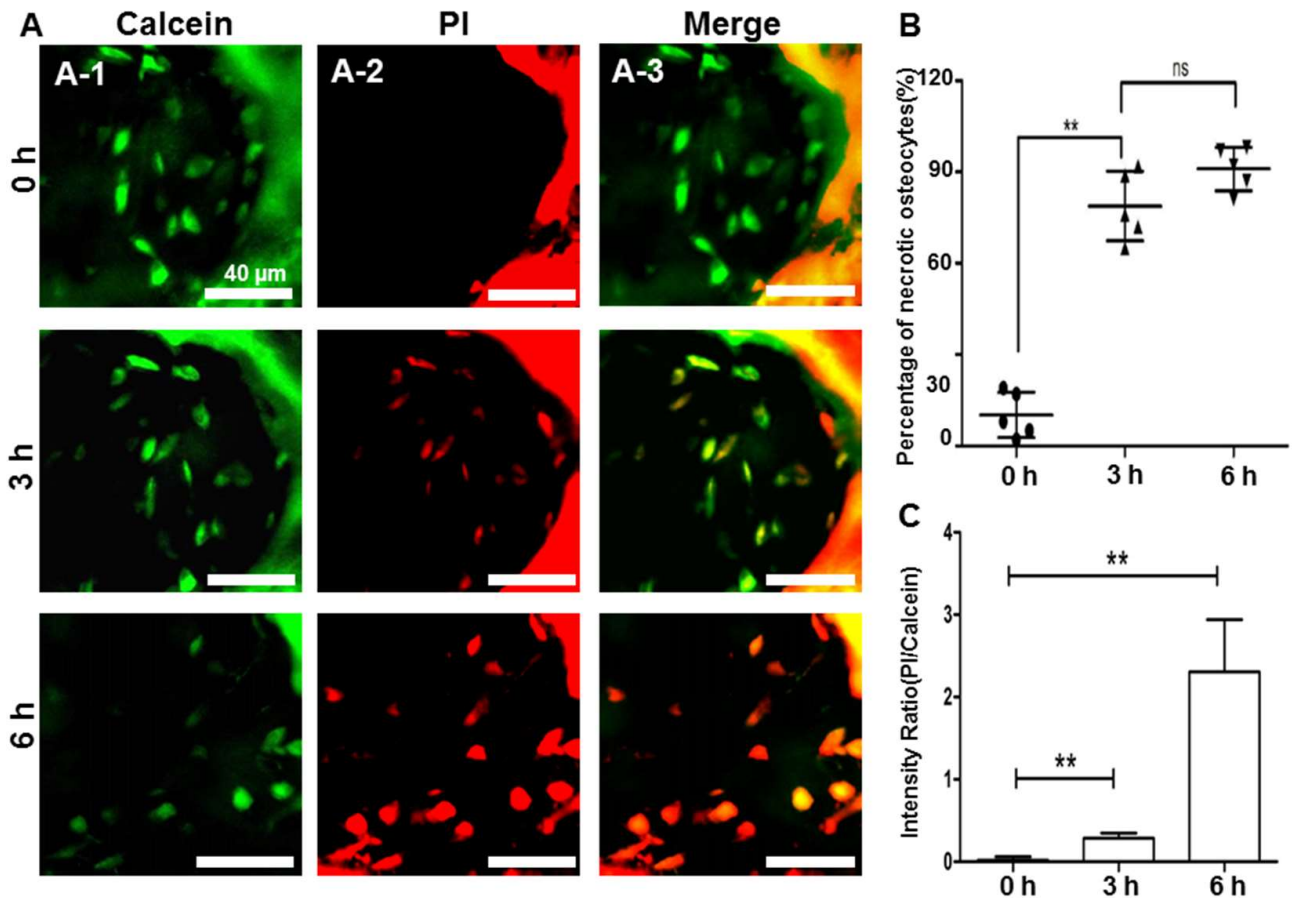

Figure 4. The changes of osteocytes after photo-thrombosis: (A) Osteocytes before they were stained with Calcein and PI at $0 \mathrm{~h} 3 \mathrm{~h}$ and $6 \mathrm{~h}$ after photo-thrombosis; (B) Percentage of dead osteocytes; (C) The intensity ratio of PI/Calcein. ${ }^{* *} p<0.01$, by a two-tailed unpaired $t$-test; ns, not significant.

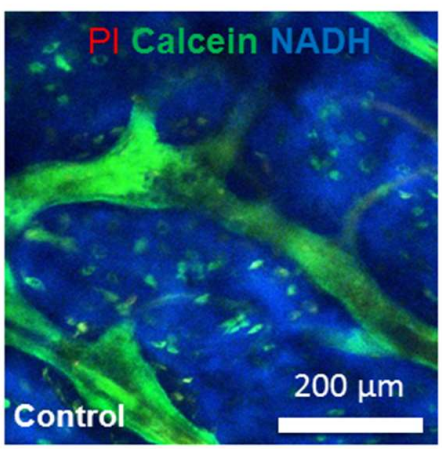

A

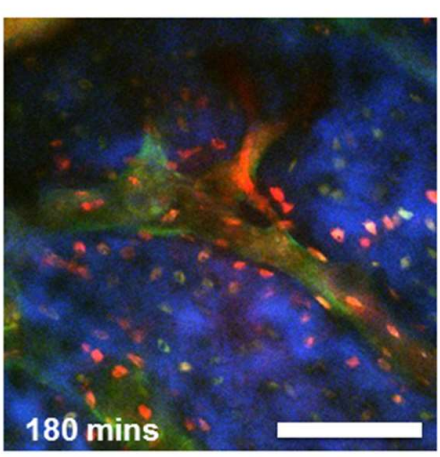

D

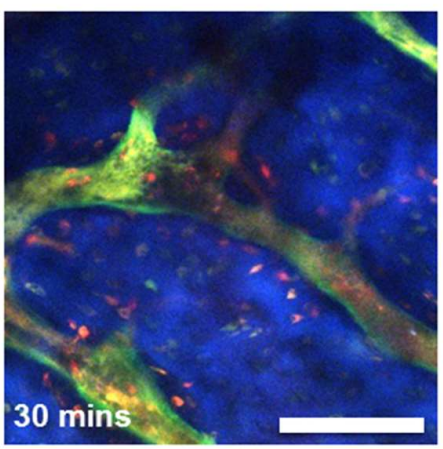

B

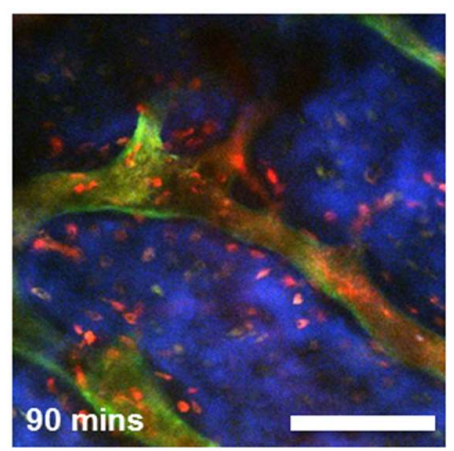

C

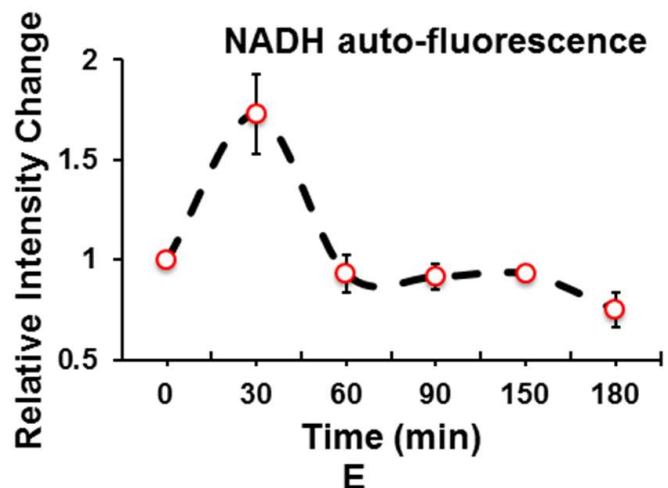

Figure 5. Intensity quantification of NADH auto-fluorescence after photo-thrombosis: (A-D) NADH auto-fluorescence (blue) changes inside the bone at the different time point (E) quantitative analysis of $\mathrm{NADH}$ auto-fluorescence change. 


\subsection{Hypoxic and Apoptotic Analysis of the Avascular Osteonecrosis Tissue}

To further verify these findings using two-photon technique, a classic AVN model was performed with transverse osteotomy. All rats survived the operation. The results showed that after osteotomy, the avascular femoral head was located in the hypoxic condition with a significant increase in HIF- $1 \alpha$ (Figure $6 \mathrm{~A}, \mathrm{C}$ ), a sensitive indicator for hypoxia. However, we did not observe apoptotic osteocytes until $6 \mathrm{~h}$ after the surgery (Figure 6 B,D), later than our observation using the photo-thrombosis model.

A

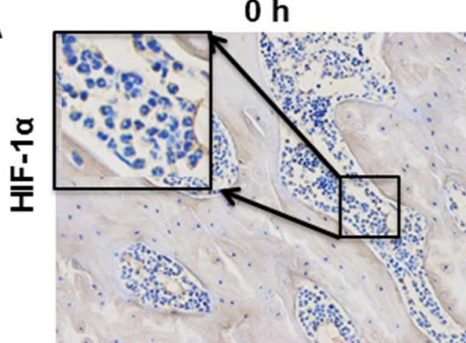

B

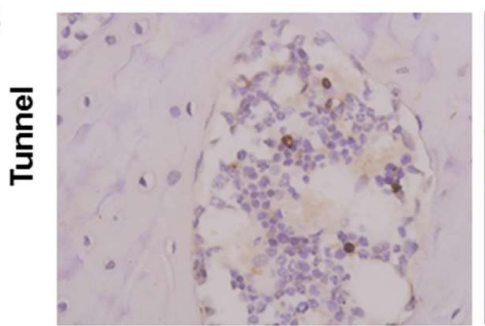

C

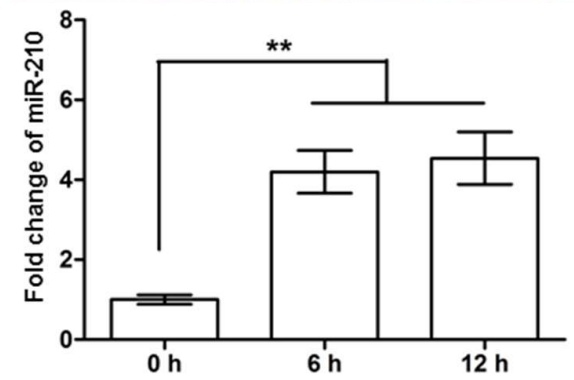

$6 \mathrm{~h}$
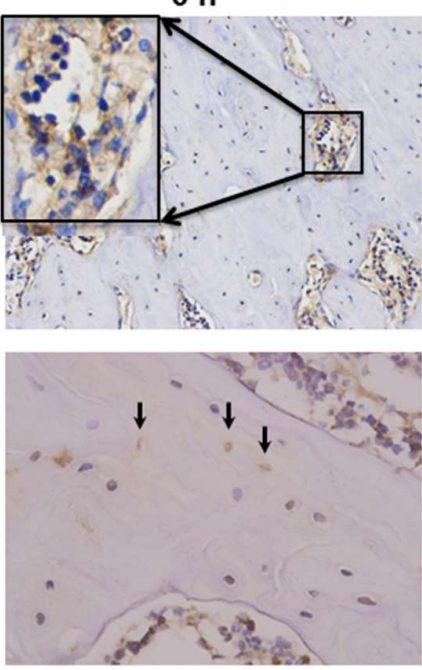

D

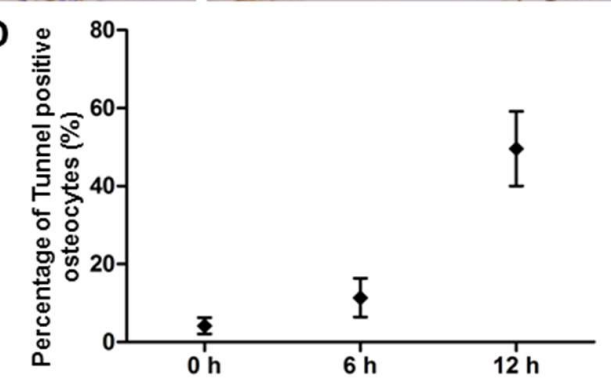

Figure 6. Histological examination of classic avascular osteonecrosis: (A) Immunohistochemical manifestation of HIF-1 $\alpha$ in different postoperative stages; (B) tunnel staining of femoral heads, the positive osteocytes are shown with black arrows; (C) HIF- $1 \alpha$ mRNA levels analyzed by real-time PCR; (D) the percentage of Tunnel positive osteocytes. ${ }^{* *} p<0.01$, by a two tailed unpaired $t$-test.

\section{Discussion and Conclusions}

The pathogenesis of AVN is complex and involves multiple cellular processes [22]. Previous studies have proposed ways to artificially induce AVN in animal models including pulsed delivery of corticosteroids $[23,24]$. However, these methods may cause side effects like thinning bones and fractures, and it is also unclear whether these models represent similar pathophysiology as AVN observed in patients. The model system used in our study overcomes these concerns through the use of a non-invasive thrombo-occlusion procedure to mimic and simulate AVN. The precise use of high intensity lasers to induce photo-thrombosis of specific arterioles enables close monitoring and tracking local responses. Recently intravital bone imaging system has been introduced to study bone homeostasis [25] and NADH was regarded as a marker in metabolism hemostasis [14,16]. Frikha-Benayed et al. also used NADH to measure metabolic oxidative stress in situ from hypoxia and found that under hypoxia (postmortem), NADH levels could be regarded as a marker to reflect metabolism change. However, they created extreme hypoxia during postmortem, which could not mimic a real hypoxic environment in a live animal and these findings were limited to the dead animals [17]. We also show that there is abundant vesselsdistributed within the bone area. 
This studyuses the photo-thrombosis approach to simulate avascular osteonecrosis (AVN) environment in live animals, and for the first time, $\mathrm{NADH}$, is used as a new metabolic marker to investigate osteocytes metabolism under the hypoxia environment in a high spatial resolution using two photon microscopy systems. Coupled with high-resolution intravital microscopy, our entire setup enabled real time tracking and monitoring of osteocyte fate and subcellular metabolic responses towards local stress signals from the ischemia within the bony environment.

Osteocytes are the terminally differentiated osteoblasts and account for over $90 \%$ of the entire cells in bone tissue [26]. The changes in osteocyte quantity and function in the setting of AVN have been investigated previously $[27,28]$. However, most studies thus far relied on the histological or ex vivo analyses of tissue or cells to extract phenotypic changes. In situ, direct observation of osteocyte response in the setting of ischemic stress is lacking. Our study showed that in real-time, osteocytes that reside within bones form an interconnected network through cytoplasmic projections [29,30], as well preferentially line the perivascular space. This observation provided strong and direct evidence that given the close proximity of osteocytes to blood vessels, avascular condition likely influences the biological activities of osteocytes.

Sato et al. reported that in a rat model with hip dislocation and osteotomy, the osteocytes death occurred at $12 \mathrm{~h}$ and AVN appeared after $96 \mathrm{~h}$ [31]. Using the similar surgical technique, we observed a few osteocytes death occurred at $6 \mathrm{~h}$ and with the majority of cell death at $12 \mathrm{~h}$, which was consistent with the previous study. However, with the photo-thrombosis technique, we observed osteocytes beginning to die at $3 \mathrm{~h}$. In this study, different from chronic formation of AVN, the high intensity stimulation in photo-thrombosis process damaged endothelial cells in the vessel rapidly. Clotting formation within the arteries was noted within seconds after initiation of laser excitation. In this context, PI rather than Tunnel assay, was used to assess for cell viability which aimed to manifest the early changes of osteocytes, which may have attributed to the early detection of osteocytes dying. In addition, we examined the calvarium and compared it to the femoral head. The differences in vascular supply as well as underlying biology between femur and calvarium bones may explain part of the differences of osteocyte responses.

NADH was an intrinsic auto-fluorescent indicator for energy metabolism [16]. In this study, ischemic and hypoxic condition inside the bone gave rise to the increase of NADH levels within $30 \mathrm{~min}$, suggesting that the disorder of energy metabolism was involved in the early pathogenesis of AVN. Within 30 min of photo-thrombosis, the blockade of electron transport chains increased the accumulation of NADH in the cell and increased fluorescence intensity. However, because photo-thrombosis is transient and reversible [32-34], reperfusion relieved the hypoxic environment and facilitated the transfer from reduced NADH state to the oxidized NAD state with no auto-fluorescence [17].

In summary, our study demonstrated that osteocyte structure and function can be imaged in vivo with two-photon microscopy by applying a combination of endogenous and exogenous fluorophores. Changes in signal can be monitored in real time after the photo-thrombosis procedure in the bone. We have shown that this technique could simultaneously facilitate qualitative examination of osteocyte morphology with detailed quantitative analysis of biological changes in cell function, which could build a more complete understanding of AVN pathophysiology in the future.

\section{Materials and Methods}

\subsection{Animal Preparation}

Six old C57BL/6J mice (6-9-month-old, male) were anesthetized by the inhalation of isoflurane ( $4 \%$ for induction; 1.5 to $2 \%$ for surgery, and 1 to $1.5 \%$ for imaging) and immobilized in a custom-made stereotactic apparatus. After anesthesia, dexamethasone and buprenorphine were subcutaneously administered to reduce inflammation and pain. Body temperature was monitored by a rectal probe and maintained at $37.0^{\circ} \mathrm{C}$ by a heating blanket (Homeothermic blanket systems, Harvard Apparatus, 
Holliston, MA, USA). Hair was removed on the skull to expose the frontoparietal skull section. Experiments were performed only if the physiological variables remained within normal limits. All experiments were performed under animal experimental guidelines approval of Mayo Clinic (A3915, 2015) and Fudan University (No. DF730, 2017).

\subsection{Bone Cell and Vasculature Labeling}

Calcein AM (Ebioscience, USA; $20 \mathrm{mg} / \mathrm{kg}$, S.C.) was injected 3 days before examination to track the live osteocytes [25]; and five min before imaging. Dextran (dextran, Texas Red, 70 kDa, Thermo Fisher Scientific., Waltham, MA, USA; $12.5 \mathrm{mg} / \mathrm{kg}$ ) was systemically administered to visualize the vasculature and blood flow in the bone.

To further characterize the damaged cells due to impaired blood supply, $100 \mu \mathrm{L}$ of a solution of propidium iodide (PI, Sigma-Aldrich, USA; $1 \mathrm{mg} / \mathrm{mL}$ ) was intravenously injected into the animal to label the dead cells.

\subsection{Multi-Photon Imaging System and Photo-Thrombosis}

The upright laser scanning microscope (BX61WI, Olympus, Tokyo, Japan) attached to a Ti:sapphire pulsed laser system ( $80 \mathrm{MHz}$ repetition rate, $<100$ fs pulse width, Spectra Physics, Santa Clara, CA, USA) and software (Fluoview1000, Olympus, Japan) was used for two-photon fluorescence imaging. $20 \times$ water immersion (NA, 1.00; WD, $2 \mathrm{~mm}$, Olympus), and $40 \times$ water-immersion objectives (NA 0.80 , WD; $3.3 \mathrm{~mm}$, Olympus) were selectively chosen for fluorescence imaging in vivo. 830-nm irradiation wavelength was used to excite calcein and dextran, and emission light was differentiated and detected with 525/50 and 615/50 filters, respectively. The average laser power for imaging was $<30 \mathrm{~mW}$.

Based on vessel diameter and blood flow direction, arterioles were discriminated and chosen as photo-thrombosis targets. System administration of Rose Bengal (RB, Sigma-Aldrich, USA; $100 \mu L$, $10 \mathrm{mg} / \mathrm{mL}$ ) was used to induce photo-thrombosis on specific arterioles under $563 \mathrm{~nm}$ laser beam illumination (intensity, $0.8-1.6 \mathrm{~mW}$ and stimulation duration, $80-100 \mathrm{~s}$ ). All the procedure finished in 15 min after RB injection.

\subsection{NADH and Extracellular Matrix Imaging}

For NADH imaging, Images were taken at 12-bit depth at a resolution of $512 \times 512$ pixels with a pixel dwell time of $4 \mu \mathrm{s}$. NADH and Texas-Red-dextran were excited at $740 \mathrm{~nm}$ and emission wavelength was separated and detected using 460/50 and 615/50 filter. In addition, due to the accumulation of minerals (calcium and phosphorus) and collagens in the bone, second harmonic generation could be used to image extracellular matrix distributions in the bone under the excitation beam at $890 \mathrm{~nm}$.

\subsection{Ischaemic Osteonecrosis Model by Osteotomy Surgery}

Fifteen male Sprague-Dawley rats were used to build up the ischaemic osteonecrosis model with transverse osteotomy. The operation was performed according to the methods used by Sato et al. [31]. The right femoral head of each rat was made surgically avascular while the contralateral side served as the control. The animals were sacrificed at different postoperative stages and the femoral heads were obtained for histological examination and hypoxic analysis (HIF-1 $\alpha$ ). The procedure for the preparation of samples can be found in our previous report [35].

\subsection{Data Analysis}

Images were processed using open source software Fiji (NIH, https: / / fiji.sc/) and an accustomed written program with MATLAB (Version 8.5.0 R2015a, Mathworks, Natick, Massachusetts, USA). Registration had been taken to perform the feature-based alignment of images at the different time point. After image registration, the number of dead osteocytes and fluorescence intensity of NADH 
were calculated or measured at five random regions of interest following background subtraction. In addition, radon transform algorithm had been used to measure blood flow velocity in the bone [36]. The data were presented as mean \pm SD if possible, and unpaired two-tailed t-test was used with a $p$ value of $<0.05$ was deemed as statistically significant.

Supplementary Materials: The following are available online.

Author Contributions: H.Y. and Y.C. and B.Y.S.K. conceived, designed and performed the experiment. H.Y., W.J. and Y.C. wrote the paper. B.Y.S.K edited and revised the paper.

Funding: This research was funded by Helen Houle Mayo Clinic Career Development Award in Neurologic Surgery (B.Y.S.K.).

Conflicts of Interest: The authors declare no conflicts of interest.

\section{References}

1. Mankin, H.J. Nontraumatic necrosis of bone (osteonecrosis). N. Engl. J. Med. 1992, 326, 1473-1479. [PubMed]

2. Chang, C.C.; Greenspan, A.; Gershwin, M.E. Osteonecrosis: Current perspectives on pathogenesis and treatment. Semin. Arthritis Rheum. 1993, 23, 47-69. [CrossRef]

3. Jergesen, H.E.; Khan, A.S. The natural history of untreated asymptomatic hips in patients who have non-traumatic osteonecrosis. J. Bone Jt. Surg. Am. 1997, 79A, 359-363. [CrossRef]

4. Yuan, H.F.; Von Roemeling, C.; Gao, H.D.; Zhang, J.; Guo, C.A.; Yan, Z.Q. Analysis of altered microRNA expression profile in the reparative interface of the femoral head with osteonecrosis. Exp. Mol. Pathol. 2015, 98, 158-163. [CrossRef] [PubMed]

5. Ragab, Y.; Emad, Y.; Abou-Zeid, A. Bone marrow edema syndromes of the hip: MRI features in different hip disorders. Clin. Rheumatol. 2008, 27, 475-482. [CrossRef] [PubMed]

6. Luk, W.H.; Au-Yeung, A.W.S.; Yang, M.K.W. Diagnostic value of SPECT versus SPECT/CT in femoral avascular necrosis: Preliminary results. Nucl. Med. Commun. 2010, 31, 958-961. [CrossRef] [PubMed]

7. Nakamura, F.; Fujioka, M.; Takahashi, K.A.; Ueshima, K.; Arai, Y.; Imahori, Y.; Itani, K.; Nishimura, T.; Kubo, T. Evaluation of the hemodynamics of the femoral head compared with the ilium, femoral neck and femoral intertrochanteric region in healthy adults: Measurement with positron emission tomography (PET). Ann. Nucl. Med. 2005, 19, 549-555. [CrossRef] [PubMed]

8. Yuan, H.F.; Shen, F.; Zhang, J.; Shi, H.C.; Gu, Y.S.; Yan, Z.Q. Predictive value of single photon emission computerized tomography and computerized tomography in osteonecrosis after femoral neck fracture: A prospective study. Int. Orthop. 2015, 39, 1417-1422. [CrossRef] [PubMed]

9. Chance, B.; Cohen, P.; Jobsis, F.; Schoener, B. Intracellular oxidation-reduction states in vivo. Science 1962, 137, 499-508. [CrossRef] [PubMed]

10. Heikal, A.A. Intracellular coenzymes as natural biomarkers for metabolic activities and mitochondrial anomalies. Biomark. Med. 2010, 4, 241-263. [CrossRef] [PubMed]

11. Obitabot, E.T.; Hanrahan, L.M.; Cachecho, R.; Beer, E.R.; Hopkins, S.R.; Chan, J.C.K.; Shapiro, J.M.; Lamorte, W.W. Changes in Hepatocyte Nadh Fluorescence during Prolonged Hypoxia. J. Surg. Res. 1993, 55, 575-580. [CrossRef] [PubMed]

12. Williamson, J.R.; Davis, K.N.; Medinaramirez, G. Quantitative-Analysis of Heterogenous Nadh Fluorescence in Perfused Rat Hearts during Hypoxia and Ischemia. J. Mol. Cell Cardiol. 1982, 14, 29-35. [CrossRef]

13. Beilke, D.; Weiss, R.; Lohr, F.; Pristovsek, P.; Hannemann, F.; Bernhardt, R.; Ruterjans, H. A new electron transport mechanism in mitochondrial steroid hydroxylase systems based on structural changes upon the reduction of adrenodoxin. Biochemistry (US) 2002, 41, 7969-7978. [CrossRef]

14. Mayevsky, A. Brain NADH redox state monitored in vivo by fiber optic surface fluorometry. Brain Res. 1984, 319, 49-68. [CrossRef]

15. Balaban, R.S.; Heineman, F.W. Control of mitochondrial respiration in the heart in vivo. Mol. Cell Biochem. 1989, 89, 191-197. [CrossRef] [PubMed]

16. Skala, M.C.; Riching, K.M.; Gendron-Fitzpatrick, A.; Eickhoff, J.; Eliceiri, K.W.; White, J.G.; Ramanujam, N. In vivo multiphoton microscopy of $\mathrm{NADH}$ and FAD redox states, fluorescence lifetimes, and cellular morphology in precancerous epithelia. Proc. Natl. Acad. Sci. USA 2007, 104, 19494-19499. [CrossRef] [PubMed] 
17. Frikha-Benayed, D.; Basta-Pljakic, J.; Majeska, R.; Schaffler, M. Regional differences in oxidative metabolism and mitochondrial activity among cortical bone osteocytes. Bone 2016, 90, 15-22. [CrossRef] [PubMed]

18. Denk, W.; Strickler, J.H.; Webb, W.W. Two-photon laser scanning fluorescence microscopy. Science 1990, 248, 73-76. [CrossRef] [PubMed]

19. Ishii, T.; Ishii, M. Intravital two-photon imaging: A versatile tool for dissecting the immune system. Ann. Rheum. Dis. 2011, 70 (Suppl. 1), i113-i115. [CrossRef] [PubMed]

20. Wang, B.G.; Konig, K.; Halbhuber, K.J. Two-photon microscopy of deep intravital tissues and its merits in clinical research. J. Microsc. 2010, 238, 1-20. [CrossRef] [PubMed]

21. Campagnola, P.J.; Loew, L.M. Second-harmonic imaging microscopy for visualizing biomolecular arrays in cells, tissues and organisms. Nat. Biotechnol. 2003, 21, 1356-1360. [CrossRef] [PubMed]

22. Assouline-Dayan, Y.; Chang, C.; Greenspan, A.; Shoenfeld, Y.; Gershwin, M.E. Pathogenesis and natural history of osteonecrosis. Semin. Arthritis Rheum. 2002, 32, 94-124. [CrossRef] [PubMed]

23. Yamamoto, T.; Irisa, T.; Sugioka, Y.; Sueishi, K. Effects of pulse methylprednisolone on bone and marrow tissues-Corticosteroid-induced osteonecrosis in rabbits. Arthritis Rheum. 1997, 40, 2055-2064. [CrossRef] [PubMed]

24. Ichiseki, T.; Kaneuji, A.; Ueda, Y.; Nakagawa, S.; Mikami, T.; Fukui, K.; Matsumoto, T. Osteonecrosis Development in a Novel Rat Model Characterized by a Single Application of Oxidative Stress. Arthritis Rheum. 2011, 63, 2138-2141. [CrossRef] [PubMed]

25. Sano, H.; Kikuta, J.; Furuya, M.; Kondo, N.; Endo, N.; Ishii, M. Intravital bone imaging by two-photon excitation microscopy to identify osteocytic osteolysis in vivo. Bone 2015, 74, 134-139. [CrossRef] [PubMed]

26. Franz-Odendaal, T.A.; Hall, B.K.; Witten, P.E. Buried alive: How osteoblasts become osteocytes. Dev. Dyn. 2006, 235, 176-190. [CrossRef] [PubMed]

27. Weinstein, R.S.; Nicholas, R.W.; Manolagas, S.C. Apoptosis of osteocytes in glucocorticoid-induced osteonecrosis of the hip. J. Clin. Endocr. Metab. 2000, 85, 2907-2912. [CrossRef] [PubMed]

28. Mutijima, E.; De Maertelaer, V.; Deprez, M.; Malaise, M.; Hauzeur, J.P. The apoptosis of osteoblasts and osteocytes in femoral head osteonecrosis: Its specificity and its distribution. Clin. Rheumatol. 2014, 33, 1791-1795. [CrossRef] [PubMed]

29. Atkins, G.J.; Findlay, D.M. Osteocyte regulation of bone mineral: A little give and take. Osteoporosis Int. 2012, 23, 2067-2079. [CrossRef] [PubMed]

30. Bonewald, L.F. The Amazing Osteocyte. J. Bone Miner. Res. 2011, 26, 229-238. [CrossRef] [PubMed]

31. Sato, M.; Sugano, N.; Ohzono, K.; Nomura, S.; Kitamura, Y.; Tsukamoto, Y.; Ogawa, S. Apoptosis and expression of stress protein (ORP150, HO1) during development of ischaemic osteonecrosis in the rat. J. Bone Jt. Surg. Br. 2001, 83, 751-759. [CrossRef]

32. Huang, A.J.W.; Watson, B.D.; Hernandez, E.; Tseng, S.C.G. Photothrombosis of Corneal Neovascularization by Intravenous Rose-Bengal and Argon-Laser Irradiation. Arch. Ophthalmol. 1988, 106, 680-685. [CrossRef] [PubMed]

33. Schroeter, M.; Jander, S.; Stoll, G. Non-invasive induction of focal cerebral ischemia in mice by photothrombosis of cortical microvessels: Characterization of inflammatory responses. J. Neurosci. Meth. 2002, 117, 43-49. [CrossRef]

34. Lee, L.J.; Yang, Z.; Rahman, M.; Ma, J.; Kwak, K.J.; McElroy, J.; Shilo, K.; Goparaju, C.; Yu, L.; Rom, W.; et al. Extracellular mRNA detected by tethered lipoplex nanoparticle biochip for lung adenocarcinoma detection. Am. J. Respir. Crit. Care Med. 2016, 193, 1431-1433. [CrossRef] [PubMed]

35. Yuan, H.F.; Pan, J.F.; Li, S.; Guo, C.A.; Liu, S.H.; Yan, Z.Q. Protective effects of total saponins of panax notoginseng on steroid-induced avascular necrosis of the femoral head in vivo and in vitro. Evid. Based Complement. Alternat. Med. 2015, 2015, 165679. [CrossRef] [PubMed]

36. Drew, P.J.; Blinder, P.; Cauwenberghs, G.; Shih, A.Y.; Kleinfeld, D. Rapid determination of particle velocity from space-time images using the Radon transform. J. Comput. Neurosci. 2010, 29, 5-11. [CrossRef] [PubMed]

Sample Availability: Not Available.

(C) 2018 by the authors. Licensee MDPI, Basel, Switzerland. This article is an open access article distributed under the terms and conditions of the Creative Commons Attribution (CC BY) license (http://creativecommons.org/licenses/by/4.0/). 